\title{
Versão portuguesa da escala de satisfação dos pacientes com os cuidados do médico de família - SatMF̧17: validação psicométrica
}

\author{
Portuguese version of the user satisfaction scale with the care of the
} family physician - SatMF17: psychometric validation

\author{
Ana Rita João1, Maria João Filomena dos Santos Pinto Monteiro², Maria da Conceição Alves Rainho Soares Pereira ${ }^{3}$, \\ Vitor Manuel Costa Pereira Rodrigues ${ }^{3} \bowtie$ \\ 1 Unidade de Saúde Familiar Corgo, ACeS Douro-1 (Marão e Douro Norte). Vila Real, Portugal. \\ Centro de Investigação em Tecnologias e Serviços de Saúde (CITSS), Escola Superior de Saúde, Universidade de Trás-os-Montes e Alto Douro (UTAD).Vila Real, \\ Portugal. \\ Centro de Investigação em Desporto, Saúde e Desenvolvimento Humano (CIDESD), Escola Superior de Saúde, Universidade de Trás-os-Montes e Alto Douro (UTAD). \\ Vila Real, Portugal.
}

Como citar este artigo (How to cite this article):

João AR, Monteiro MJFSP, Pereira MCARS, Rodrigues VMCP. Versão portuguesa da escala de satisfação dos pacientes com os cuidados do médico de família - SatMF17: validação psicométrica (Portuguese version of the user satisfaction scale with the care of the family physician - SatMF17: psychometric validation). Sci Med. 2018;28(3):ID31286. DOI: 10.15448/1980-6108.2018.3.31286

\section{RESUMO}

OBJETIVOS: Avaliar as propriedades psicométricas da escala de satisfação dos pacientes com os cuidados do médico de família (SatMF17). MÉTODOS: Estudo metodológico, em que participaram usuários inscritos em Unidades de Saúde Familiar e Unidades de Cuidados de Saúde Personalizados do Agrupamento de Centros de Saúde Marão e Douro Norte. Os critérios de inclusão dos usuários foram: ser paciente inscrito numa das duas unidades referenciadas, ter mais de 18 anos e menos de 65 anos, saber ler e escrever e ter tido, pelo menos no último ano, uma consulta em uma das duas unidades. A escala SatMF17 é constituída por 17 itens, respondidos por meio de uma escala tipo Likert que varia entre 1 (mau) e 5 (excelente), em que o somatório varia entre 17 e 85 . A coleta dos dados foi realizada no período compreendido entre janeiro e junho de 2016. O paciente que preenchesse os critérios de inclusão quando da chegada à unidade de saúde era convidado a participar do estudo pelo assistente administrativo. Após apresentação e explicação dos objetivos do estudo e assinatura do termo de consentimento, era oferecido o questionário ao paciente, que ao final do autopreenchimento o inseria em um envelope. Para análise dos dados foram utilizados os recursos estatísticos coeficiente alfa de Cronbach, análise fatorial exploratória, critérios de Kaiser-Meyer-Olkin, teste de esfericidade de Bartlett e coeficiente de correlação de Pearson.

RESULTADOS: A amostra incluída foi de 284 pacientes, sendo 159 (56,0\%) participantes do gênero feminino. A média de idade situou-se nos 41,2 anos, variando entre 18 anos e 65 anos. A análise fatorial exploratória em componentes principais e rotação varimax revelou uma solução fatorial de dois fatores com autovalores superiores a 1, os quais explicam em conjunto $72,48 \%$ da variância total da satisfação dos pacientes com os cuidados do médico de família.

CONCLUSÕES: A escala SatMF17 é confiável e válida e pode ser utilizada em populações com características semelhantes, constituindo um instrumento de utilidade prática para avaliar a satisfação dos pacientes com os cuidados do médico de família.

DESCRITORES: satisfação do paciente; clínicos gerais; estudos de validação.

\section{ABSTRACT}

AIMS: To evaluate the psychometric properties of the satisfaction scale of patients with the care of the family physician (SatMF17).

METHODS: Methodological study, in which users enrolled in Family Health Units and Personalized Health Care Units of the Marão and Douro Norte Health Centers. The criteria for inclusion of the users were: to be a patient enrolled in one of the two referenced units, to be over 18 and under 65 years old, to be able to read and write and to have had, at least, one visit in the last year in one of the two units. The SatMF17 scale consists of 17 items, answered by a Likert scale ranging from 1 (bad) to 5 (excellent), in which the sum ranges from 17 to 85 . The data collection was carried out in the period between January and June 2016. The patient who met the inclusion criteria upon arrival at the health unit was invited to participate in the study by the administrative assistant. After a presentation and explanation of the objectives of the study, and signing of the consent term, the questionnaire was offered to the patient, who at the end of self-completion inserted it into an envelope. For statistical analysis, the statistical resources were Cronbach's alpha coefficient, exploratory factorial analysis, Kaiser-Meyer-Olkin criteria, Bartlett's sphericity test and Pearson's correlation coefficient.

RESULTS: The sample included had 284 patients, of whom $159(56,0 \%)$ were female. The mean age was 41,2 years, ranging from 18 years to 65 years. The exploratory factorial analysis in main components and varimax rotation revealed a factorial solution of two factors with eigenvalues greater than 1 , which together explain $72,48 \%$ of the total variance of patient satisfaction with the care of the family doctor. CONCLUSIONS: The SatMF17 Scale is reliable and valid and can be used in populations with similar characteristics, constituting an instrument of practical utility to evaluate the satisfaction of patients with the care of the family doctor.

KEYWORDS: patient satisfaction; general practitioners; validation studies. 
Abreviaturas: ACeS, Agrupamento de Centros de Saúde; AFE, Análise Fatorial Exploratória; ARC, Atitudes Relacionais e Comportamentais; IA, Informação e Apoio; SatMF17, escala de satisfação dos pacientes com os cuidados do médico de família.

\section{INTRODUÇÃO}

De uma forma geral, para determinar a qualidade de um determinado bem ou serviço é necessário caracterizar e medir o nível de satisfação na perspetiva dos clientes. O objetivo primordial de qualquer organização de saúde é assegurar a qualidade e segurança dos cuidados que disponibiliza aos cidadãos. Porém, não deve apenas limitar-se à produção de um serviço ou produto de qualidade, mas também satisfazer o cliente, motivar a procura do serviço e, de preferência, levar o cliente a escolher este em função de outro. Um serviço que é avaliado externamente e satisfaz as necessidades dos seus clientes é, em princípio, um serviço com qualidade [1].

Quando as expectativas e necessidades dos pacientes estão satisfeitas, verifica-se uma perceção mais positiva e maior possibilidade de os resultados serem alcançados [2]. Baixas expectativas em relação ao serviço disponibilizado estão geralmente associadas a maior satisfação, assim como expectativas mais elevadas podem associar-se a menor satisfação com o serviço. Um estudo desenvolvido em 31 países da União Europeia, incluindo Portugal, com o objetivo de avaliar a influência dos indicadores socioeconômicos (gênero, educação, rendimento familiar e etnicidade) na satisfação dos pacientes nos cuidados de saúde primários, confirmou achados prévios de altos níveis de satisfação com os cuidados de saúde primários na Europa. Esse estudo verificou que cerca de $75 \%$ da satisfação era explicada pelo perfil do paciente, sendo que as mulheres, grupos com baixos rendimentos familiares e a primeira geração de imigrantes eram os menos satisfeitos. Por outro lado, mostrou que cuidados centrados na pessoa refletem maior satisfação [3]. Ferreira [4] e Freitas et al. [5] afirmaram que a satisfação do paciente envolve avaliação pessoal relativamente aos serviços de saúde prestados, constituindo um juízo sobre a qualidade dos cuidados de saúde, com base nos seus valores, experiências, expectativas e percepções. Paula et al. [6] salientam aspectos relacionados ao contexto em que ocorre a experiência com os serviços de saúde como fator que influencia a opinião em termos de satisfação global com esses serviços.
Há evidência de que a satisfação dos pacientes, além de ser um indicador importante da qualidade dos serviços, influencia muitos dos comportamentos e resultados de saúde. Ademais, pacientes mais satisfeitos com os cuidados estão mais predispostos a cumprir as prescrições e tratamentos médicos e a manter-se na mesma equipe de saúde, permitindo a consolidação da relação terapêutica e a melhora do seu estado de saúde [7,8].

A preocupação com a avaliação da satisfação levou a que desde os anos 1970 venham sendo utilizado instrumentos para medir a satisfação do paciente, os quais têm acompanhando as conjecturas relativas ao significado de satisfação [9]. A avaliação da satisfação dos clientes é um parâmetro de avaliação dos cuidados e uma forma de envolver os pacientes na prestação de cuidados, pois estes são o mais importante em qualquer sistema de saúde [10].

No presente estudo, pretende-se avaliar as propriedades psicométricas da escala de satisfação dos pacientes com os cuidados do médico de família (SatMF17), que integra o instrumento desenvolvido pelo Europep [11].

\section{MÉTODOS}

\section{Delineamento, amostra e aspectos éticos}

Foi realizado um estudo metodológico que pretendeu analisar as propriedades psicométricas da escala de avaliação SatMF17 para a sua validação. A seleção da amostra obedeceu aos seguintes critérios: ser usuário inscrito nas unidades Saúde Familiar e Unidades de Cuidados de Saúde Personalizados do Agrupamento de Centros de Saúde (ACeS) Marão e Douro Norte, ter mais de 18 e menos de 65 anos, ter tido pelo menos um contato com a unidade funcional no último ano e saber ler e escrever.

A escala SatMF17 é um instrumento de autopreenchimento que integra um questionário mais amplo desenvolvido pelo Europep [11]. A escala SatMF17 é constituída por 17 itens, respondidos por meio de uma escala tipo Likert que varia entre 1 (mau) e 5 (excelente), em que o somatório varia entre 17 e 85 . Pontuações superiores indicam maior satisfação com os cuidados do médico de família. Os questionários eram considerados válidos se os usuários respondessem a pelo menos 14 dos 17 itens.

O projeto foi aprovado pela Comissão de Ética para a Saúde da Administração Regional de Saúde do Norte (parecer no 105/2015) e autorizado pela Direção Executiva do Agrupamento dos Centros de Saúde Marão e Douro Norte. Foram assegurados o 
anonimato e a confidencialidade das informações coletadas [12].

\section{Coleta de dados}

A coleta dos dados foi realizada no período compreendido entre janeiro e junho de 2016. Procedeu-se à reunião com os coordenadores das respetivas unidades funcionais para apresentação do estudo e operacionalização dos procedimentos. Acordou-se que o questionário e o consentimento informado seria disponibilizado pelo assistente administrativo, após treinamento prévio.

O paciente que preenchesse os critérios de inclusão quando da chegada à unidade de saúde era convidado a participar do estudo pelo assistente administrativo. Após breve apresentação e explicação dos objetivos do estudo e salvaguardada a confidencialidade e anonimato do participante, este era informado de que poderia desistir a qualquer momento sem que ocorresse qualquer consequência. Era disponibilizado o consentimento informado que, após assinatura, era recolhido pelo assistente administrativo. Só após este procedimento era oferecido o questionário ao paciente, que ao final do autopreenchimento o inseria em um envelope.

\section{Análise estatística}

Para analisar as características psicométricas da escala SatMF17, recorreu-se à estatística descritiva (medidas de tendência central e frequências absolutas e relativas) e análise multivariada. No tratamento estatístico foi utilizado o suporte informático IBM SPSS Statistics 24. A fidelidade da escala foi avaliada por meio do coeficiente alfa de Cronbach, que mede a consistência interna da escala. Para analisar a validade de construto efetuou-se a Análise Fatorial Exploratória (AFE) por meio da Análise dos Componentes Principais, recorrendo à rotação ortogonal, segundo o método Varimax. A análise fatorial exploratória permite encontrar uma estrutura fatorial que explique as correlações entre os itens da escala, visando a identificar o construto latente, especificamente em relação à sua dimensionalidade, tornando possível ajustar a escala à amostra em estudo [13,5]. A adequação dos dados para utilização desta análise foi verificada pelos critérios de Kaiser-Meyer-Olkin e pelo teste de esfericidade de Bartlett. Os critérios definidos para a seleção de fatores na AFE foram: i) valor próprio superior a 1 (eigenvalues $>1$ ); ii) fidelidade individual dos itens $(\lambda)$, sendo o valor de referência o peso fatorial superior a 0,50 , podendo considerar-se superior a 0,40 , o que ocorreu neste estudo [14]; iii) cada fator deve explicar no mínimo 5\% da variância [15]. Seguiu-se a análise correlacional em que foram analisadas as correlações entre itens da escala, bem como entre estes e o fator, por meio do coeficiente de correlação de Pearson.

\section{RESULTADOS}

Do total de 298 participantes, foram excluídos 14 por terem preenchido menos de 14 entre as 17 questões da escala SatMF17, obtendo-se um total de 284 questionários válidos. A amostra ficou então constituída por 284 participantes, sendo $159(56,0 \%)$ do gênero feminino. A média de idade situou-se nos $41,2 \pm 12,9$ anos, variando entre 18 anos e 65 anos. As habilitações acadêmicas, o estado civil e situação profissional dos participantes estão descritos na Tabela 1.

Os usuários recorreram a uma das unidades

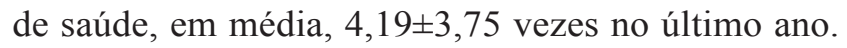
A consulta programada foi procurada em $79,9 \%$ das vezes, enquanto $20,1 \%$ corresponderam à consulta aberta, que é a situação que ocorre em caso de doença aguda, que necessita atendimento no próprio dia.

Tabela 1. Caracterização da amostra quanto às variáveis sociodemográficas. Agrupamento de Centros de Saúde Marão e Douro Norte, 2016

\begin{tabular}{lcc}
\hline Característica & $\mathbf{n}$ & $\%$ \\
\hline Habilitação acadêmica & & \\
Sabe ler e escrever & 13 & 4,6 \\
10 Ciclo do Ensino Básico & 38 & 13,4 \\
2o Ciclo Ensino Básico & 35 & 12,3 \\
3o Ciclo de Ensino Básico & 44 & 15,5 \\
Ensino Secundário & 84 & 29,6 \\
Ensino Médio & 8 & 2,8 \\
Ensino Superior & 59 & 20,8 \\
Não respondeu & 3 & 1,1 \\
Estado civil & & \\
Casado & 154 & 54,2 \\
Solteiro & 98 & 34,5 \\
Divorciado & 14 & 4,9 \\
Viúvo & 7 & 2,5 \\
Separado & 3 & 1,1 \\
Não respondeu & 8 & 2,8 \\
Situação profissional & & \\
Ativo & 166 & 58,5 \\
Desempregado & 71 & 25,0 \\
Aposentado & 16 & 5,6 \\
Estudante & 15 & 5,3 \\
Outra & 12 & 4,2 \\
Não respondeu & 4 & 1,4 \\
\hline
\end{tabular}




\section{Análise Fatorial Exploratória}

$\mathrm{Na}$ AFE da escala foram utilizados determinados procedimentos estatísticos. No sentido de prosseguir com a análise fatorial da escala SatMF17, previamente foi aplicado o critério de Kaiser-Meyer-Olkin, que permitiu verificar a adequação dos dados à amostra, obtendo-se um valor de 0,95 , considerado um valor muito bom [14]. O teste de Esfericidade de Bartlett apresentou valores $\chi^{2}=4811,74 ;(p<0,001)$, que permitiu rejeitar a hipótese nula e confirmar a utilização do método de análise para os dados colhidos. A AFE em componentes principais e rotação varimax revelou uma solução fatorial de dois fatores com autovalores superiores a 1 , os quais explicam, em conjunto, $72,48 \%$ da variância total da satisfação dos pacientes com os cuidados do médico de família, sendo o primeiro fator responsável por $38,87 \%$ e o segundo fator por $33,61 \%$. Os valores encontrados são considerados como acima do mínimo aceitável [14, 15] (Tabela 2).

Os resultados da análise da AFE mostram que no Fator 1 saturaram nove itens, designados para a subescala Atitudes Relacionais e Comportamentais (ARC) $(3,4,5,6,7,8,11,12$, e 14). No Fator 2 saturaram oito itens na subescala Informação e Apoio (IA) (9, 10, $16,17,18$ e 19). Verifica-se que, para todos os itens, a saturação ocorre nos respectivos fatores e, dado que os valores fatoriais são positivos e superiores a 0,5 , consideramos que os fatores ARC e IA contribuem para o construto satisfação dos pacientes com os cuidados do médico de família. Posteriormente, determinou-se a consistência interna da escala, apresentando um alpha de Cronbach de 0,960, o que evidencia consistência interna muito alta da SatMF17. Os coeficientes de $\alpha$ de Cronbach para cada fator foram $\mathrm{ARC}=0,955 \mathrm{e}$ IA $=0,930$, mostrando valores de consistência interna muito altos [16].

\section{Estudo Correlacional}

A correlação entre cada item e destes com o respectivo fator mostrou para o Fator 1 (subescala ARC) correlações moderadas e altas, variando entre 0,472 e 0,883 [17]. As correlações entre os itens e o Fator 1 apresentaram pontuações altas, exceto para o item 8 "Confidencialidade da informação sobre o seu processo" que foi moderada $(0,690)$, como consta na Tabela 3.

Tabela 2. Matriz das cargas fatoriais da escala de satisfação dos pacientes com os cuidados do médico de família - SatMF17. Agrupamento de Centros de Saúde Marão e Douro Norte, 2016

\begin{tabular}{|c|c|c|}
\hline \multirow{2}{*}{ Item / descrição } & \multicolumn{2}{|c|}{ Componentes } \\
\hline & Fator 1 (ARC) & Fator 2 (IA) \\
\hline 4. Interesse mostrado pela sua situação pessoal & 0,879 & \\
\hline 7. Forma como o médico o/a ouviu & 0,873 & \\
\hline 5. Facilidade com que se sentiu à vontade para Ihe contar os seus problemas & 0,852 & \\
\hline 3. Fazê-lo/a sentir que tem tempo durante a consulta & 0,827 & \\
\hline 6. Forma como foi envolvido/a nas decisões sobre os cuidados que o médico prestou & 0,808 & \\
\hline 11. Atenção dispensada aos seus problemas & 0,716 & \\
\hline 12. Exame clínico feito pelo médico & 0,701 & \\
\hline 8. Confidencialidade da informação sobre o seu processo & 0,690 & \\
\hline 14. Explicação sobre medicamentos, tratamentos e exames prescritos & 0,609 & \\
\hline 17. Apoio que recebeu para compreender porque é importante seguir os conselhos do seu médico & & 0,811 \\
\hline $\begin{array}{l}\text { 18. Conhecimento sobre o que foi feito e dito em anteriores contatos nesta Unidade de Saúde Familiar/Unidade } \\
\text { de Cuidados de Saúde Personalizados }\end{array}$ & & 0,804 \\
\hline 9. Forma como lhe foi prestado o alívio rápido dos sintomas & & 0,798 \\
\hline 16. Ajuda que recebeu para enfrentar os problemas emocionais relacionados com o seu estado de saúde & & 0,766 \\
\hline 10. Ajuda que recebeu para se sentir suficientemente bem para desempenhar as suas tarefas diárias & & 0,759 \\
\hline $\begin{array}{l}\text { 19. Preparação sobre o que esperar dos cuidados hospitalares, de outros especialistas ou outros prestadores } \\
\text { de cuidados }\end{array}$ & & 0,746 \\
\hline 15. Forma como foi suficientemente informado/a sobre sintomas e a sua doença & & 0,632 \\
\hline 13. Oferta de serviços de prevenção de doenças (por exemplo, rastreio, pedido de exames, vacinas) & & 0,586 \\
\hline
\end{tabular}

ARC, Atitudes Relacionais e Comportamentais; IA, Informação e Apoio. 
Tabela 3. Matriz de correlação inter-itens e a subescala Atitudes Relacionais e Comportamentais (ARC, fator 1). Agrupamento de Centros de Saúde Marão e Douro Norte, 2016

\begin{tabular}{|c|c|c|c|c|c|c|c|c|c|c|}
\hline Item (Fator1) & Fator 1 & Item 4 & Item 7 & Item 5 & Item 3 & Item 6 & Item 11 & Item 12 & Item 8 & Item 14 \\
\hline Fator1 & 1 & & & & & & & & & \\
\hline Interesse mostrado pela sua situação pessoal (Item 4) & $0,919 * *$ & 1 & & & & & & & & \\
\hline Forma como o médico o/a ouviu (Item 7) & $0,894^{* *}$ & $0,882^{* *}$ & 1 & & & & & & & \\
\hline $\begin{array}{l}\text { Facilidade com que se sentiu à vontade para lhe contar os } \\
\text { seus problemas (Item 5) }\end{array}$ & $0,821^{* *}$ & $0,813^{* *}$ & $0,791^{* *}$ & 1 & & & & & & \\
\hline Fazê-lo/a sentir que tem tempo durante a consulta (Item 3) & $0,877^{* *}$ & $0,840^{* *}$ & $0,811^{* *}$ & $0,743^{* *}$ & 1 & & & & & \\
\hline $\begin{array}{l}\text { Forma como foi envolvido/a nas decisões sobre os } \\
\text { cuidados que o médico lhe prestou (Item 6) }\end{array}$ & $0,891 * *$ & $0,839^{* *}$ & $0,883^{* *}$ & $0,773^{* *}$ & $0,791^{* *}$ & 1 & & & & \\
\hline Atenção dispensada aos seus problemas (Item 11) & $0,885^{* *}$ & $0,766^{* *}$ & $0,698^{* *}$ & $0,668^{* *}$ & $0,708^{* *}$ & $0,700^{* *}$ & 1 & & & \\
\hline Exame clínico feito pelo médico (Item 12) & $0,883^{* *}$ & $0,743^{* *}$ & $0,733^{* *}$ & $0,624^{* *}$ & $0,708^{* *}$ & $0,776^{* *}$ & $0,761^{* *}$ & 1 & & \\
\hline Confidencialidade da informação (Item 8) & $0,690^{* *}$ & $0,600^{* *}$ & $0,604^{* *}$ & $0,705^{* *}$ & $0,583^{* *}$ & $0,595^{* *}$ & $0,479 * *$ & $0,562 * *$ & 1 & \\
\hline $\begin{array}{l}\text { Explicação sobre medicamentos, tratamentos e exames } \\
\text { prescritos (Item 14) }\end{array}$ & $0,802^{* *}$ & $0,678^{* *}$ & $0,622^{* *}$ & $0,580^{* *}$ & $0,643^{* *}$ & $0,618^{* *}$ & $0,687^{* *}$ & $0,765^{* *}$ & $0,472^{* *}$ & 1 \\
\hline
\end{tabular}

A correlação entre cada item e destes com o respectivo fator mostrou para o Fator 2 (subescala IA) correlações moderadas e altas, variando entre 0,466 e 0,760 [17]. As correlações entre os itens e o Fator 2 apresentaram pontuações altas, como consta na Tabela 4.

Como a validade de construto é determinada pela grandeza dos valores das cargas fatoriais e como os resultados são superiores a 0,5 [18], podemos afirmar que a escala SatMF17, composta de dois fatores com um total de 17 itens, apresenta validade de construto.

Da observação das correlações entre cada item e o respectivo fator pode-se afirmar que os itens medem o fator, dado que apresenta correlações significativas e elevadas para um nível de significância de $\mathrm{p}<0,01$.

\section{DISCUSSÃO}

Decorrente da necessidade de se dispor de instrumentos válidos e confiáveis, este estudo teve como objetivo avaliar as propriedades psicométricas da SatMF17. Os estudos realizados por outros autores, nomeadamente os desenvolvidos em Portugal, não procederam à AFE das dimensões da SatMF17 [11]. Após a análise fatorial exploratória confirmou-se estrutura composta por dois fatores devidamente individualizados e que explicam no seu conjunto $72,48 \%$ da variância total do construto satisfação dos pacientes com os cuidados do médico de família. Perante os resultados verificados, o modelo bifatorial exposto da SatMF17 mostra evidência empírica para sua utilização, designadamente no que respeita à validade do construto [15].

Tabela 4. Matriz de correlação inter-itens e a subescala Informação e Apoio (IA, fator 2). Agrupamento de Centros de Saúde Marão e Douro Norte, 2016

\begin{tabular}{|c|c|c|c|c|c|c|c|c|c|}
\hline Item (Fator2) & Fator 2 & Item 9 & Item 10 & Item 17 & Item 18 & Item 19 & Item 16 & Item 13 & Item 15 \\
\hline Fator2 & 1 & & & & & & & & \\
\hline Forma como lhe foi prestado o alívio rápido dos sintomas (Item 9) & $0,821 * *$ & 1 & & & & & & & \\
\hline $\begin{array}{l}\text { Ajuda que recebeu para se sentir suficientemente bem para } \\
\text { desempenhar as suas tarefas diárias (Item 10) }\end{array}$ & $0,820^{* *}$ & 0,726 & 1 & & & & & & \\
\hline $\begin{array}{l}\text { Apoio que recebeu para compreender porque é importante seguir os } \\
\text { conselhos do seu médico (Item 17) }\end{array}$ & $0,791^{* *}$ & 0,615 & 0,659 & 1 & & & & & \\
\hline $\begin{array}{l}\text { Conhecimento sobre o que foi feito e dito em anteriores contactos } \\
\text { (Item 18) }\end{array}$ & $0,883^{* *}$ & 0,705 & 0,665 & 0,730 & 1 & & & & \\
\hline Preparação sobre o que esperar dos cuidados (Item 19) & $0,818^{* *}$ & 0,626 & 0,586 & 0,637 & 0,749 & 1 & & & \\
\hline Ajuda que recebeu para enfrentar os problemas (Item 16) & $0,773^{* *}$ & 0,588 & 0,611 & 0,760 & 0,678 & 0,535 & 1 & & \\
\hline Oferta de serviços de prevenção de doenças (Item 13) & $0,799 * *$ & 0,559 & 0,600 & 0,539 & 0,645 & 0,594 & 0,466 & 1 & \\
\hline Forma como foi informado/a sobre sintomas e a sua doença (Item 15) & $0,839 * *$ & 0,612 & 0,571 & 0,641 & 0,677 & 0,657 & 0,582 & 0,712 & 1 \\
\hline
\end{tabular}


Quanto ao tamanho da amostra importa referir que reúne os requisitos para os procedimentos da AFE, recomendados por Marôco [18], que propõem entre cinco a dez participantes por item do instrumento (17 itens), sendo que neste estudo foram recolhidos 284 questionários válidos. Dado que esta escala e os fatores que a integram apresentam valores de consistência interna muito elevados, os itens que a constituem medem de forma robusta o construto em estudo. Em outras palavras, quanto maior é a qualidade das ARC bem como a informação e apoio disponibilizados, melhor é a perceção de satisfação dos pacientes com os cuidados do médico de família.

É importante destacar que o Plano Nacional de Saúde de Portugal, revisão e extensão 2020, apresenta os seguintes eixos estratégicos transversais: cidadania, equidade e acesso, qualidade em saúde e políticas de saúde [19]. O plano incentiva a multiplicação de mecanismos de participação do cidadão no setor da saúde, preconizando novas metodologias para avaliar a satisfação dos pacientes com os cuidados de saúde, para além da tradicional caixa de reclamações e opiniões e do livro de reclamações. A satisfação do cliente é um indicador fundamental para monitorar a qualidade em saúde, especificamente para os cuidados disponibilizados pelo médico de família. Esta problemática está bem reforçada no Plano Nacional de Saúde 2015-2020, em que o cidadão é "entendido como central no sistema de saúde, é um importante agente de participação e de mudança no desempenho de diferentes papéis, e o de consumidor com expectativas e direito a cuidados seguros e de qualidade" [19].

Os resultados do presente estudo indicam que se deve continuar a avaliar a dimensionalidade do instrumento, sendo importante assinalar algumas limitações, que também foram descritas no estudo de Freitas [5], como o fato de os dados terem sido coletados em uma amostra não probabilística. Logo, não se pode afirmar a representatividade da amostra em virtude de não se ter comparado o perfil da amostra com o perfil da população, nem tampouco generalizar as conclusões. Tal como recomenda Freitas [5], em futuros estudos devem ser analisadas a validade convergente e discriminante e a estabilidade temporal. A escala SatMF17, apesar de ter mostrado uma adequada validade de construto, requer a sua aplicação no âmbito de estudos longitudinais, com amostras probabilísticas de usuários inscritos em unidades de saúde familiar.

Apesar destas limitações, concluiu-se que a escala SatMF17 apresenta adequada validade de construto e boas propriedades psicométricas e pode constituir um instrumento de utilidade prática para avaliar a satisfação dos pacientes com os cuidados do médico de família. Ela pode ser utilizada em populações com características semelhantes, e os dados obtidos podem contribuir para a operacionalização das estratégias do Plano Nacional de Saúde.

Este instrumento evidenciou um modelo bifatorial, composto por ARC e IA. Observou-se que o questionário foi de fácil preenchimento pelos participantes e contribui para a avaliação da satisfação e, deste modo, permite a concepção e implementação de estratégias que visem à melhoria da satisfação dos pacientes com os cuidados do médico de família.

\section{NOTAS}

Apoio financeiro

Este estudo não recebeu apoio financeiro de fontes externas.

Declaração de conflito de interesses

Os autores declaram não haver conflitos de interesses relevantes ao conteúdo deste estudo.

Contribuições dos autores

Todos os autores fizeram contribuições substanciais para concepção, ou delineamento, ou aquisição, ou análise ou interpretação de dados; e redação do trabalho ou revisão crítica; e aprovação final da versão para publicação.

Disponibilidade dos dados e responsabilidade pelos resultados

Todos os autores declaram ter tido total acesso aos dados obtidos e assumem completa responsabilidade pela integridade destes resultados.

\section{REFERÊNCIAS}

1. Batista R. Qualidade percebida pelos utentes das IPSS de Oliveira do Hospital. Dissertação [dissertation]. [Aveiro]: Universidade de Aveiro; 2010. 99p.

2. Hollanda E, Siqueira SA, Andrade GR, Molinaro A, Vaitsman J. Satisfação e responsividade em serviços de atenção à saúde da Fundação Oswaldo Cruz. Ciênc. Saúde Coletiva. 2012;17(12):3343-52. https://doi.org/10.1590/S141381232012001200019

3. Detollenaere J, Hanssens L, Schäfer W, Willems S. Can you recommend me a good GP? Describing social differences in patient satisfaction within 31 countries. Int J Qual Health Care. 2018;30(1):9-15. https://doi.org/10.1093/intqhc/mzx157 
4. Ferreira PL. Avaliação dos doentes de cuidados primários: Enquadramento conceptual. Rev Port Clin Geral. 2000; 16:53-62.

5. Freitas MJ, Parreira P, Domingues JP. Avaliação das propriedades psicométricas da Escala Satisfação dos Clientes com os Cuidados de Enfermagem no Hospital. Rev Enf Ref. 2016;IV(10):9-17. https://doi.org/10.12707/RIV16031

6. Paula A, Long R, Wiener D. Are Your Patients Satisfied?. Mark Health Serv. 2002;22(3):28-32.

7. Fernandes, ME. Avaliação da satisfação de utentes de um serviço prestador de cuidados de saúde mental comunitários: estudo exploratório. Dissertação [dissertation]. [Porto], Universidade Católica Portuguesa, 2014. 47p.

8. Di Matteo MR, Giordani PJ, Lepper HS, Croghan TW. Patient adherence and medical treatment outcomes: a metaanalysis. Med Care. 2002;40(9):794-811. https://doi.org/10.1097/00005650-200209000-00009

9. Gill L, White L. A critical review of patient satisfaction. Leadership Health Serv. 2009;22(1):8-19. https://doi. org/10.1108/17511870910927994

10. Biscaia A, Martins J, Carreira M, Gonçalves I. Cuidados de Saúde Primários em Portugal: reformar para novos sucessos. Lisboa: Padrões Conceptuais Editora; 2006.

11. Roque H, Veloso A, Ferreira PL. Versão portuguesa do questionário EUROPEP: contributos para a validação psicométrica. Rev Saúde Pública. 2016;50:61. https://doi.org/10.1590/S1518-8787.2016050006259

12. Fortin MF. Fundamentos e etapas do processo de investigação. Lisboa: Lusodidata; 2009.

13. Marôco JP. Análise Estatística com o SPSS Statistics. 5ª edição. Pêro Pinheiro: ReportNumber; 2011.

14. Marôco JP. Análise Estatística com o SPSS Statistics. 6a edição. Pêro Pinheiro: ReportNumber; 2014.

15. Pereira RP, Guerra AC, Cardoso MJ, Santos AT, Figueiredo MC, Carneiro AC. Validação da versão portuguesa do Questionário de Eficácia Clínica e Prática Baseada em Evidências. Rev Latino-Am Enfermagem. 2015;23(2):345-51. https://doi.org/10.1590/0104-1169.0367.2561

16. Marôco JP, Garcia-Marques T. Qual a fiabilidade do alfa de Cronbach? Questões antigas e soluções modernas? Laboratório de Psicologia. 2006;4(1):65-90.

17. Pestana MH, Gageiro JN. Análise de dados para ciências sociais: a complementaridade do SPSS. $5^{\mathrm{a}}$ edição. Lisboa: Edições Sílabo; 2008.

18. Marôco JP. Análise de Equações Estruturais: Fundamentos teóricos, Software e Aplicações. Pêro Pinheiro: ReportNumber; 2010.

19. Portugal. Minitério da Saúde. Direção Geral da Saúde. Plano nacional de saúde: revisão e extensão a 2020. Ministério da Saúde [Internet]. Lisboa, 2015. p.14. Available from: http://1nj5ms21li5hdggbe 3mm7ms5.wpengine.netdna-cdn.com/ files/2015/06/Plano-Nacional-de-Saude-Revisao-e-Extensao-a-2020.pdf $€$ 